\title{
La acción del Estado brasileño en relación a los migrantes bolivianos en Brasil: la cuestión del trabajo (in)documentado, refugio e inmigración económica ${ }^{\star}$
}

\author{
The action of the Brazilian State regarding Bolivian migrants in Brazil: \\ the issue of (un)documented labor, refuge and economic immigration
}

\author{
Sonia Ranincheski** \\ Roberto Rodolfo Georg Uebel ${ }^{* * *}$ \\ Universidad Federal do Rio Grande do Sul, Porto Alegre, Brasil
}

Recibido: 20 de enero de 2014. Aprobado: 13 de agosto de 2014.

\begin{abstract}
Resumen
Desde inicios del siglo XXI Brasil ha estado inserto en una agenda internacional de desarrollo y crecimiento económico, presentando buenas condiciones laborales y de proyección global, representando un puerto de ingreso de variados grupos inmigratorios, incluyendo a los bolivianos, que se insertan cada vez más en la sociedad y economía brasileña - muchas veces ilegalmente- e influenciando el papel del Estado y las relaciones diplomáticas entre el país emisor y el receptor de estos inmigrantes. Esta investigación abordará la acción del Estado brasileño y la inserción de los inmigrantes bolivianos en Brasil por medio de un análisis bibliográfico, documental y estadístico, además de inferir las puntualidades de esta inmigración en las relaciones Brasil-Bolivia y establecer proyecciones para las dos primeras décadas del nuevo siglo.
\end{abstract}

Palabras claves: inmigración, bolivianos, Brasil.

\footnotetext{
El presente artículo forma parte de las investigaciones que se desarrollaron en el marco del Proyecto $\mathrm{N}^{\circ} 25136$ "Interesses, valores e ações estratégicas das elites latino-americanas e a relação com os Estados Unidos e Europa", financiado por la Universidad Federal do Rio de Grande Sul.

* Profesora e investigadora en el curso de Relaciones Internacionales de la Universidad Federal do Rio Grande do Sul, UFRGS en Porto Alegre, Rio Grande del Sur, Brasil. Correo electrónico: sonia.ranincheski@ufrgs.br

*** Actualmente es estudiante de Maestría en Geografía de la Universidad Federal do Rio Grande do Sul, UFRGS, estudiante de Especialización en Administración Pública de la Universidad Federal de Santa Maria, UFSM e investigador del Laboratorio del Espacio Social, UFRGS y del Laboratorio de Estudios Internacionales, UFSM. Correo electrónico: roberto.uebel@ufrgs.br
} 


\begin{abstract}
From the beginning of the $21^{\text {st }}$ Century, Brazil has been inserted in an agenda of international development and economic growth, presenting good labor conditions and global projections, representing a port of entry for various immigrant groups, including Bolivians, who are increasingly inserted into Brazilian society and economy-frequently illegally-and have influenced the role of the State and the diplomatic relationship between the country of origin and country of arrival of these immigrants. Hence, this article will deal with the insertion of Bolivian immigrants in Brazil through bibliographical, documental and statistical analysis, and will conclude on the specificities of this immigration on the Brazil-Bolivia relations and the projections for the first two decades of the new century.
\end{abstract}

Keywords: immigration, bolivians, Brazil.

\title{
Introducción
}

El siglo XXI se ha caracterizado como una nueva "era de la migración" (Castles, Miller y Ammendola, 2005), alcanzando el mismo nivel en ese período del siglo XIX y principios del siglo XX. La migración se refiere a la movilización de personas o poblaciones enteras de un país a otro y está directamente relacionada con la cuestión del Estado nacional, de las fronteras nacionales, y las capacidades de los Estados para absorber y retener miles de nuevos individuos que ingresan en sus espacios. Están involucrados otros temas que tensionan política y socialmente a los Estados tales como las condiciones de soberanía y territorialidad en el actual sistema internacional y la posición política del Estado en la administración de la inmigración. La complejidad del tema de inmigración permite, incluso, que algunos autores manifiesten la existencia de otras conexiones, como las redes sociales, los migrantes culturales, políticos y económicos - en el sentido más amplio del término- que no necesariamente se ajustan a la rigidez de los límites geográficos y nacional de los Estados burocráticos (Castles, 2004). Este artículo se concentra en analizar el papel del Estado y sus capacidades o condiciones de enfrentar el tema de la inmigración. En específico, tratamos del Estado brasileño y la creciente inmigración de bolivianos hacia este país. 
La sociedad brasileña se ha caracterizado por la fuerte presencia del Estado en los diversos temas de la vida social, sea privada o pública. Tal es la fuerza del aparato del Estado que muchos autores, con razón, destacan que en el Brasil, en primer lugar, a lo largo de los siglos se formó el Estado y sus instituciones más elementales para después formar la sociedad civil. En este sentido, es posible acercarse al tema de la inmigración considerando las condiciones y características del Estado en abordar la migración.

Además, es pertinente estudiar la inmigración en cuanto a los aspectos mismos de las poblaciones que migran (los hechos que hacen este proceso de migración), las sociedades que reciben los migrantes (las diversas formas de integración), y las instancias legales de este tránsito de personas (los acuerdos migratorios externos e internos entre los países). En otras palabras, se trata de verificar en los procesos de migración las cuestiones sociales, nacionales y estatales.

En este artículo discutimos el ámbito de la acción del Estado brasileño y sus capacidades de manejar el fenómeno de la inmigración de bolivianos al país, que puede estar relacionada, entre otras razones, a cuestiones de trabajo, a las condiciones sociales y políticas bolivianas y, en algunos casos, al tráfico de drogas.

Una de las explicaciones ponderadas por la literatura para la elección del Brasil, llevando en consideración las tres razones citadas, está relacionada a las buenas condiciones económicas de Brasil, en comparación con la situación en Bolivia, que despiertan en los bolivianos el sueño de una vida mejor, a pesar de la diferencia cultural entre los dos países. Conforme afirma Sayad (1999), pese a las evidencias contrarias, el inmigrante solamente existe para el trabajo, por el trabajo y en el trabajo. Afuera del trabajo él no tiene salvación.

La permanencia de los inmigrantes bolivianos en suelo brasileño está concentrada en las regiones fronterizas y en grandes ciudades, sobre todo en São Paulo, donde ellos enfrentan realidades adversas marcadas por la falta de documentos y por la discriminación racial y social. No obstante, en las estadísticas presentadas por el Ministerio del Trabajo y Empleo (MTE) brasileño, desde 2010, los únicos Estados brasileños que presentan inmigrantes bolivianos con autorizaciones de trabajo son Acre, Ceará y Pará. Cabe destacar que son estadísticas oficiales y por lo tanto, no están incluidas en ellas las personas que entran al país y no tienen registro, no tienen documentos, los llamados indocumentados (Tabla 1). 
Tabla 1

Número y situación de los ciudadanos bolivianos en el Brasil (2008-2013)

\begin{tabular}{lrrrrrrr}
\hline Clasificación / Año & 2008 & 2009 & 2010 & 2011 & 2012 & $2013^{*}$ & Total \\
\hline Fronterizos & 18 & 92 & 32 & 171 & 26 & 15 & 354 \\
Otra clasificación & 2 & 0 & 0 & 1 & 0 & 0 & 3 \\
Permanentes & 1.904 & 13.304 & 2.349 & 1.720 & 1.020 & 222 & 20.519 \\
Provisionales & 350 & 4.591 & 409 & 53 & 4 & 2 & 5.409 \\
$\begin{array}{l}\text { Autorizaciones } \\
\text { temporales }\end{array}$ & 336 & 1.158 & 1.520 & 10.873 & 15.696 & 4.277 & 33.860 \\
Refugiados & 0 & 52 & 5 & 4 & 4 & 0 & 65 \\
\hline Total & 2.610 & 19.197 & 4.315 & 12.822 & 16.750 & 4.516 & 60.210 \\
\hline
\end{tabular}

Fuente: Datos provenientes de documentos obtenidos por medio de la Ley de Acceso a la Información. ( ${ }^{*}$ Hasta septiembre de 2013).

La situación de los bolivianos sin documentos, sus pésimas condiciones de vida y la frágil política de inmigración por parte del Estado brasileño, forman parte de un fenómeno paradojal: estos inmigrantes buscan mejores condiciones de vida en el Brasil y encuentran condiciones muchas veces peores que aquellas que tenían en el país de origen, permaneciendo en ellos el sentimiento de frustración frente a la realidad que viven.

Precisamente, el objetivo del artículo es tratar el tema de los bolivianos en Brasil presentando datos y reflexionar sobre la acción del Estado brasileño. Se considera que la definición del Estado como una asociación de dominación con carácter institucional ha tratado, con éxito, de monopolizar dentro de un territorio la violencia física legítima como medio de dominación. Con este fin, ha reunido todos los medios materiales en manos de sus dirigentes y ha expropiado a todos los funcionarios estamentales que antes disponían de ellos por derecho propio, sustituyéndolos con sus propias jerarquías supremas (Weber, 2004).

En este marco teórico sobre el Estado, una vez presentadas las acciones o las inexistencias de estas acciones del Estado brasileño, intentamos demostrar que Brasil está inserto en el tema de la inmigración en masa. Además de eso, pretendemos mostrar la fragilidad y la ausencia de política para la inmigración. Brasil presentó un desarrollo económico y una estabilidad en su economía en los últimos 20 años; el Estado está sufriendo nuevas funciones históricas y está incorporando nuevos conceptos condicionados de las 
variables mundiales (Schmidt, 2013, p. 179). Trabajamos, entonces, con la hipótesis de que el Estado brasileño aún no está preparado para recibir la cantidad de inmigrantes que ha llegado en la última década y para ello usamos como metodología el análisis histórico y las estadísticas oficiales del gobierno brasileño. El artículo está estructurado para problematizar el tema del Estado y sus capacidades para generar una política de inmigración, en general, y, en específico, el caso de la inmigración boliviana. En la primera parte presentamos la discusión sobre el papel histórico del Estado brasileño. En la segunda parte hacemos una reflexión sobre la llegada de los bolivianos, la caracterización de esta migración en el Brasil. En la tercera parte discutimos la situación degradante de vida de los bolivianos, las condiciones inhumanas de trabajo, casi en una situación de esclavitud. Finalmente, tratamos los refugiados políticos y las cuestiones culturales de los bolivianos en Brasil.

\section{El papel del Estado brasileño ante la inmigración boliviana}

No se puede describir y entender el fenómeno migratorio sin que se comprenda la categoría de Estado nacional en la esfera política de un marco administrativo de poder que confiere nacionalidad y en la esfera mental del inmigrado que se obliga a pensar sobre esta cuestión, en muchos casos, por primera vez (Sayad, 1999). La cuestión es relevante una vez que la implementación del Estado moderno tiene como punto de apoyo la centralización del poder, a partir de múltiples unidades de control territorial por medios militares y tributarios, así como el establecimiento de supremacía de un derecho universal objetivo sobre todos los intereses y derechos particulares. El Estado occidental ha sido construido históricamente en la tensión entre las aspiraciones individuales de personas o grupos de interés y las propuestas de soporte colectivo o clases sociales (Schmidt, 2013, p. 190). El Estado brasileño, en particular, fue edificado, para resolver estas tensiones, en una mezcla de dominación tradicional y estamental, si seguimos los tres tipos weberiano de Estado (2002, p. 198). Los poderes particulares de los grupos dominantes se combinan con el administrativo estatal, cuyos funcionarios adquieren una importancia y un dominio en el aparato del Estado. La burocracia brasileña fue importante para el fortalecimiento del Estado central en Brasil, simultáneamente se torna casi propietario de decisiones estatales. Estas características se modificarán lentamente en dirección a un Estado más centrado en leyes, normas y procedimientos conocidos y seguidos por todos. En este sentido, mirar las normas que se formarán en el tema de la inmigración puede ser una manera de tratar el tema de la inmigración de bolivianos y el Estado brasileño. 
Adoptando la Convención $N^{\circ} 143$ de la Organización Internacional del Trabajo, cuyo artículo $1^{\circ}$ establece que "todo Miembro para cual la presente Convención está en vigor se compromete a respetar los derechos humanos fundamentales de todos los trabajadores migrantes" [traducción de los autores] (Ministério do Trabalho e Emprego, 2008), el Brasil da un paso para que no haya discriminación entre brasileños y extranjeros en materia de empleo. Con esta ratificación, el Brasil parece señalar un camino opuesto a los de países que han creado medidas discriminatorias en relación a los trabajadores migrantes. El Consejo Nacional de Inmigración del Brasil, en su sexta reunión plenaria de 2008, ha aprobado una Moción de Repudio a la nueva Directiva de la Unión Europea sobre el trato a los migrantes. En el texto del informe final, el Consejo Nacional de Inmigración del Brasil (CNIg) refuerza la cuestión:

Teniendo conocimiento de la edición de una nueva norma migratoria por la Unión Europea que prevé el endurecimiento del trato de los trabajadores migrantes que viven en sus países miembros, estableciendo incluso la detención por tiempo prolongado de adultos y niños, se aprueba, por unanimidad, un repudio a esa normativa por considerarse que viola los derechos fundamentales de migrantes en cuanto personas humanas y trabajadores, constituyendo una medida discriminatoria que está, claramente, en contradicción a la histórica receptividad del Brasil a los inmigrantes procedentes del continente europeo [traducción de los autores] (Conselho da Comunidade Luso-Brasileira do Estado de São Paulo, 2008).

En Europa las políticas de inmigración son diferenciadas. Estas pueden caracterizarse en tres modelos. El primer modelo, integracionista, adoptado por Francia, cuyo punto central es defender el respeto a las leyes del país, independiente de las diferencias culturales que los inmigrantes traigan consigo. El segundo modelo, multiculturalista, utilizado por los ingleses, defiende que no hay relación entre los diferentes grupos que llegan a Inglaterra y los ingleses. En otras palabras, se construye una realidad de guetos culturales para los inmigrantes; estos, obviamente, deben respetar las leyes del país. Finalmente, el tercer modelo es el intercultural, aplicado en España y en Cataluña, en especial, cuyas características apuntan a una política de aceptación de los inmigrantes que llegan con sus diversidades culturales y la posibilidad de la diversidad religiosa, dando ciertas garantía a los ciudadanos para practicar su religión y tener educación y salud universal.

Una de estas prácticas adoptadas por el Estado brasileño durante los últimos años es el monitoreo de los movimientos en las fronteras y la construcción de políticas públicas regionales de integración, promoviendo, por primera vez, una cierta ampliación del debate sobre la cuestión de las migraciones en el ámbito del MERCOSUR (Almeida, 
2008a). Otra de las iniciativas es la fijación del Día Internacional de los Inmigrantes, siguiendo la orientación de la Organización de las Naciones Unidas, que definió el 18 de diciembre de 1990 como fecha conmemorativa y adoptó la Convención Internacional sobre la Protección de los Derechos de todos los Trabajadores Migrantes y de los Miembros y sus Familias. Otra acción es el ingreso del Brasil, en 2005, en la Organización Internacional para las Migraciones (OIM).

De los ministerios del Estado brasileño, tres se destacan por la constante actuación, además de dos subórganos fundamentales para la amalgamación del trabajo y actividad inmigrante en el Brasil. El Ministerio de la Seguridad Social, por ejemplo, realizó seminarios y editó los resultados de las discusiones ocurridas en ellos, apuntando, según los organizadores de la obra, que:

Así como cabe al Estado diseñar los mecanismos de regulación para que los flujos financieros, de mercado y de conocimiento contribuyan al desarrollo nacional, también cabe a ello establecer instrumentos que permitan que las migraciones de trabajadores ocurran sin que estos trabajadores pierdan su protección social y sus derechos de seguridad social. En ambos casos, el establecimiento de reglas claras y la garantía de derechos parece ser la condición necesaria para el bienestar de las personas y el progreso de los países [traducción de los autores] (Ministério da Previdência Social, 2006).

El más activo de los ministerios brasileños, en relación a la situación de los trabajadores inmigrantes, es, hasta el momento, el Ministerio del Trabajo y Empleo (MTE), que creó y gerencia la Coordinación General de Inmigración, CGIg, desde el 2004, además de mantener el Consejo Nacional de Inmigración, CNIg, creado en 1980 y en funcionamiento hasta el presente. Es el CNIg el que brinda los procedimientos para la autorización del trabajo a extranjeros, y establece otros parámetros legales en relación al trabajo de los inmigrantes (Conselho Nacional de Imigração, 1997). La CGIg es la unidad administrativa del MTE que tiene la competencia sobre la decisión respecto a las solicitudes de autorización de trabajo a extranjeros, mediante la presentación del requerimiento conforme el "Formulario de Requerimiento de Autorización de Trabajo", firmado y conducido por su representante legal.

Con funciones diferenciadas, dichas unidades de este Ministerio parecen presentar datos contradictorios, que necesitan ser relativizados y revisados. Sin embargo, para efecto de construcción de este artículo, consideramos los datos de la CGIg, toda vez que es la responsable operacional por la cuestión del trabajo inmigrante. Siendo así, una de las informaciones para el caso de los inmigrantes bolivianos está en la Tabla 2, justamente 
por su ausencia. ¿Cómo explicar esta situación? ¿Los bolivianos no solicitan visas o los visados solicitados son reprobados?

Tabla 2

Autorizaciones de trabajo concedidas por país de origen (2010-2013)

\begin{tabular}{|c|c|c|c|c|}
\hline País & 2010 & 2011 & 2012 & $2013^{*}$ \\
\hline EE.UU. & 7.520 & 10.098 & 9.138 & 4.021 \\
\hline Reino Unido & 3.828 & 2.500 & 4.363 & 2.320 \\
\hline Filipinas & 6.530 & 7.798 & 5.179 & 2.056 \\
\hline Alemania & 2.873 & 3.162 & 3.589 & 1.639 \\
\hline Portugal & 757 & 1.547 & 2.171 & 1.474 \\
\hline España & 1.425 & 1.844 & 1.992 & 1.453 \\
\hline India & 3.234 & 4.262 & 4.235 & 1.390 \\
\hline Japón & 1.761 & 2.260 & 2.316 & 1.367 \\
\hline China & 2.154 & 2.639 & 3.082 & 1.207 \\
\hline Italia & 2.006 & 2.421 & 2.999 & 1.169 \\
\hline Francia & 1.597 & 2.166 & 2.369 & 1.085 \\
\hline Países Bajos & 1.137 & 1.222 & 1.337 & 753 \\
\hline Canadá & 836 & 1.170 & 1.167 & 648 \\
\hline Corea del Sur & 897 & 1.440 & 2.311 & 636 \\
\hline Noruega & 1.925 & 1.821 & 1.316 & 633 \\
\hline Polonia & 884 & 1.044 & 942 & 541 \\
\hline México & 774 & 1.059 & 1.246 & 485 \\
\hline Venezuela & 547 & 768 & 525 & 443 \\
\hline Indonesia & 1.819 & 2.682 & 2.306 & 438 \\
\hline Rusia & 578 & 919 & 842 & 362 \\
\hline Ucrania & 610 & 641 & 774 & 320 \\
\hline Grecia & 463 & 410 & 556 & 305 \\
\hline Rumania & 628 & 750 & 698 & 275 \\
\hline Croacia & 408 & 581 & 625 & 266 \\
\hline Malasia & 254 & 327 & 346 & 259 \\
\hline Suecia & 446 & 469 & 533 & 255 \\
\hline
\end{tabular}




\begin{tabular}{lrrrr}
\hline & & & & \\
Bélgica & 399 & 534 & 642 & 238 \\
Dinamarca & 459 & 641 & 537 & 216 \\
Colombia & 1.068 & 1.014 & 1.010 & 214 \\
Finlandia & 213 & 236 & 307 & 187 \\
Otros & 7.441 & 10.652 & 7.767 & 2.831 \\
\hline Total & 55.471 & 69.077 & 67.220 & 29.486 \\
\hline
\end{tabular}

Fuente: Datos provenientes de documentos obtenidos por medio de la Ley de Acceso a la Información. Actualizado al 30/06/2013 ( ${ }^{*}$ Primer semestre 2013).

Hay un gran interés en el extranjero por trabajar en el Brasil. Probablemente, una de las razones de este interés es la economía brasileña en expansión durante las últimas dos décadas y el uso de equipamientos y tecnología extranjera que exigen mano de obra calificada, poco frecuente en el Brasil. Así, las compañías extranjeras traen de otros países a profesionales expertos que se quedan por un período determinado. Este proceso no es desconocido para las autoridades brasileñas que, para regular y uniformizar los procedimientos de análisis de las solicitudes de autorizaciones de trabajo extranjero de las empresas internacionales, han creado el orden de servicio/GM/CNIg/Número 01/11, que actualiza la resolución de la normativa número 61/2004 del Consejo Nacional de Inmigración.

El contingente de trabajadores de Sudamérica y del MERCOSUR que demanda atención por parte del Estado brasileño ha comenzado a tener reglas específicas a partir de 2008, con la reglamentación del ingreso de los trabajadores sudamericanos en el país, con un trato preferente para aquellos que se establecen de manera formal (Conselho Nacional de Imigração, 2008).

De acuerdo con el coordinador general de Inmigración del Ministerio del Trabajo y Empleo (MTE), Paulo Sérgio de Almeida, "la idea es facilitar la obtención de la visa de trabajo para que las personas no ingresen de forma irregular en el territorio brasileño y no sean explotadas y sometidas al trabajo esclavo" [traducción de los autores] (Almeida, 2008b).

Los datos de la Policía Federal brasileña son significativos en este sentido. En septiembre de 2013 existían aproximadamente 177.000 inmigrantes sudamericanos - entre regulares e irregulares- en el territorio brasileño. Independientemente que el flujo de inmigrantes es grande, todavía no hay un mecanismo que reglamente esta inmigración 
en masa. Cabe destacar, sin embargo, los límites del alcance de esta reglamentación, la cual fija reglas para que el MTE autorice el trabajo de personas extranjeras y controle el flujo migratorio en Brasil, pero no impide que se utilice el medio más usado para amparar a los inmigrantes extranjeros trabajadores: recurrir a acuerdos internacionales o al matrimonio "funcional" con un brasileño. En otras palabras, Brasil todavía no tenía en 2013 una norma específica que exigiese un cambio en su legislación.

La inmigración de trabajadores en Sudamérica y especialmente en dirección al Brasil obliga a los gobiernos brasileños a establecer una posición tanto en las situaciones domésticas como internacionales, incluyendo acuerdos con otros países, como es el caso del acuerdo con Bolivia, firmado en 2005 y renovado en 2006 y 2007, conforme a datos del Ministerio de Relaciones Exteriores del Brasil. Con este acuerdo entra en vigencia el Instrumento Ejecutivo entre el Gobierno de la República Federativa del Brasil, el Gobierno de la República de Bolivia y el Escritorio Regional para el Cono Sur de la OIM, con el propósito de promover la integración socioeconómica de los nacionales de los dos países que se encuentran en una situación inmigratoria irregular en el territorio de sus relacionamientos amigables existentes (Ministério das Relações Exteriores, 2008).

Es válido destacar la actuación del Ministerio de Justicia del Brasil, por medio de su Departamento de Extranjeros y del Departamento de Policía Federal, en el área de la actividad inmigrante, a través de la elaboración de estadísticas, control, fiscalización y acompañamiento de las actividades sociolaborales de los inmigrantes bolivianos en el territorio brasileño, en especial con un trabajo en conjunto con el Ministerio Público Federal y secretarías estatales y municipales de Justicia vis-à-vis la exploración del trabajo inmigrante ilegal por compañías brasileñas.

El Instituto Brasileño de Geografía y Estadística (IBGE) también trabaja en el control de los grupos inmigratorios por medio de la realización de estadísticas de inferencia y localización de estos grandes grupos, resaltando los últimos dos censos, en la presentación de los resultados, en virtud de la composición de los inmigrantes en la sociedad y en el desarrollo socioeconómico brasileño. En especial es válido resaltar que en el Censo de 2000 había, según el IBGE, 11.113 ciudadanos declarados bolivianos en el territorio brasileño, número que se elevó a 27.259 en el Censo de 2010, según datos del Instituto Brasileiro de Geografia e Estatística, más que el doble de ciudadanos registrados en el censo anterior (datos provenientes de documentos obtenidos por medio de la Ley de Acceso a la Información). 
El Estado brasileño viene realizando acciones en el ámbito internacional que llaman la atención hacia el tema de la inmigración y de las condiciones del trabajo, pero están lejos de sanar los males por los cuales los inmigrantes pasan. De esta manera, la actuación de los órganos gubernamentales brasileños empieza a ser requerida cada vez con más frecuencia y firmeza, en vista de la defensa de los derechos de los inmigrantes, bien como su acceso a las infraestructuras básicas del Estado brasileño. Cabe señalar que,

no obstante, la existencia de una legislación específica referente a los extranjeros aún en disonancia en relación a la garantía de los derechos fundamentales de determinados inmigrantes en Brasil, la Constitución Federal brasileña de 1988 posibilitó el acceso universal de cualquiera persona al sistema de salud, sea nacional o extranjera, sea un inmigrante documentado o indocumentado [traducción de los autores] (Waldman, 2011, p. 108).

La paradoja en este caso es que existe una ley que permite a los inmigrantes el acceso al sistema de salud, aunque no exista una política pública empleada por el Estado brasileño para la promoción de la salud dirigida a comunidades de inmigrantes residentes en São Paulo y en otros sitios del Brasil. De ahí, como afirma Silva (1999), las redes migratorias formadas entre los que se van y los que permanecen en el país de origen son imprescindibles cuando se habla del tema de migraciones internacionales (Waldman, 2011). En cuanto a los accesos a infraestructuras básicas brasileñas, es una realidad de muchas de las comunidades fronterizas del Brasil con países latinoamericanos, que tiene una dinámica propia, en la cual las personas no brasileñas buscan auxilio sobre todo en temas como la salud (DalPrá, Mendes y Mioto, 2007).

Otras entidades de la sociedad civil actúan frente al problema, pero sin articulación precisa. Podemos citar el Sindicato de los Trabajadores del Comercio de São Paulo, Sindicato de las Costureras de São Paulo y Osasco, Asociación de los Abogados del Trabajo, Consulado General de Corea del Sur, Consulado General de Bolivia, Asociación Brasileña de Coreanos, Cámara de Comercio e Industria Brasil-Corea, Asociación Bolivia-Brasil (BolBra), Centro de Apoyo al Migrante, Pastoral del Migrante, Asociación de los Confeccionistas del Bom Retiro y Sindicato de la Industria del Vestuario.

Estas entidades de la sociedad civil participaron en 2008, según el MTE, de 13 grandes reuniones en la capital paulista. El resultado fue la creación del "Pacto Municipal de Tres Partes Contra el Fraude y la Precarización, por el Empleo y Trabajo Decentes en São Paulo". El pacto es una propuesta de entidades lideradas por la Superintendencia Regional del Trabajo y Empleo del Estado de São Paulo (SRTE/SP) para combatir la precarización del trabajo de los inmigrantes extranjeros en la capital, sobre todo aquella 
asociada a la actividad textil ejercida por bolivianos y coreanos, en acuerdo con el MTE (Camargo, 2008).

En palabras de la jefa de Fiscalización de la SRTE/SP, Ana Palmira Arruda Camargo, por el pacto, "las empresas, oficinas y confecciones, bien como asociaciones y representantes de los migrantes, se comprometen a buscar la regularización de las situaciones de forma asistida y orientada" (Camargo, 2008). Se trata de pasar al nivel del:

proceso en si ha hecho con que esa comunidad pasase a ver la actuación del Ministerio del Trabajo y Empleo de forma positiva y adversa del papel de la policía, pasando a encarar nosotros como parejas en la legalización y defensa de sus derechos y no más como una entidad represora (Camargo, 2008).

Las acciones citadas son políticamente relevantes para el gobierno brasileño porque señalan que el Estado no está inerte en relación al problema. Sin embargo, representan solo una parte del proceso de combate de la inmigración irregular y del trabajo, si no son acompañadas por medidas eficaces de control y fiscalización, principalmente de los empleadores brasileños.

\section{La llegada de los bolivianos}

¿Cómo llegan los inmigrantes bolivianos a Brasil? La frontera todavía es una alternativa muy utilizada. Las puertas de ingreso para el Brasil son las ciudades de Corumbá (Estado del Mato Grosso do Sul), Cáceres (Estado del Mato Grosso), Guajará-Mirim (Estado del Amazonas, por vía fluvial), Manaus (Estado del Amazonas también por vía fluvial) y más recientemente Foz do Iguaçu (en el Estado del Paraná, limítrofe con el Paraguay), donde ingresan sin documentos por medio del Puente de la Amistad, conforme muestra el Mapa 1. 


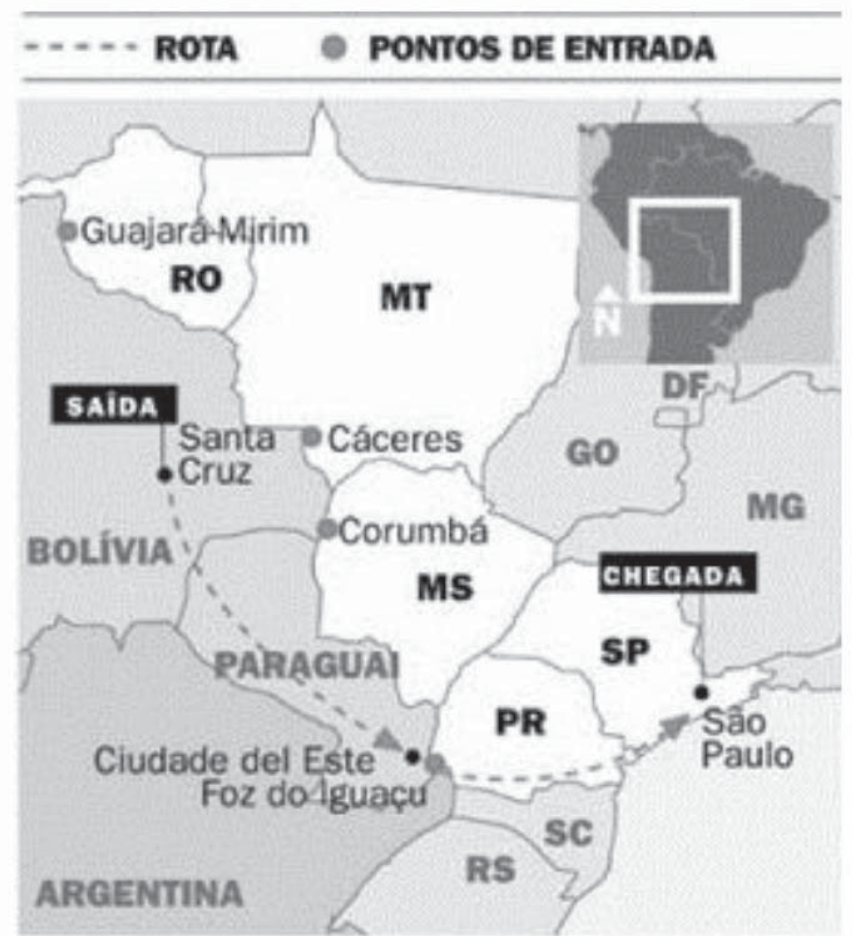

Mapa 1. Puntos y rutas de ingreso de inmigrantes bolivianos en teritorrio brasileño (en portugués). Fuente: Souchaud y Baeninger, 2008.

Cuando llegan a Brasil, vía frontera, muchos de ellos se quedan en las cercanías, como se observa en el Mapa 2 y en la Tabla 3. Luego, la gran mayoría se traslada hasta la ciudad de São Paulo (Souchaud y Baeninger, 2008). 


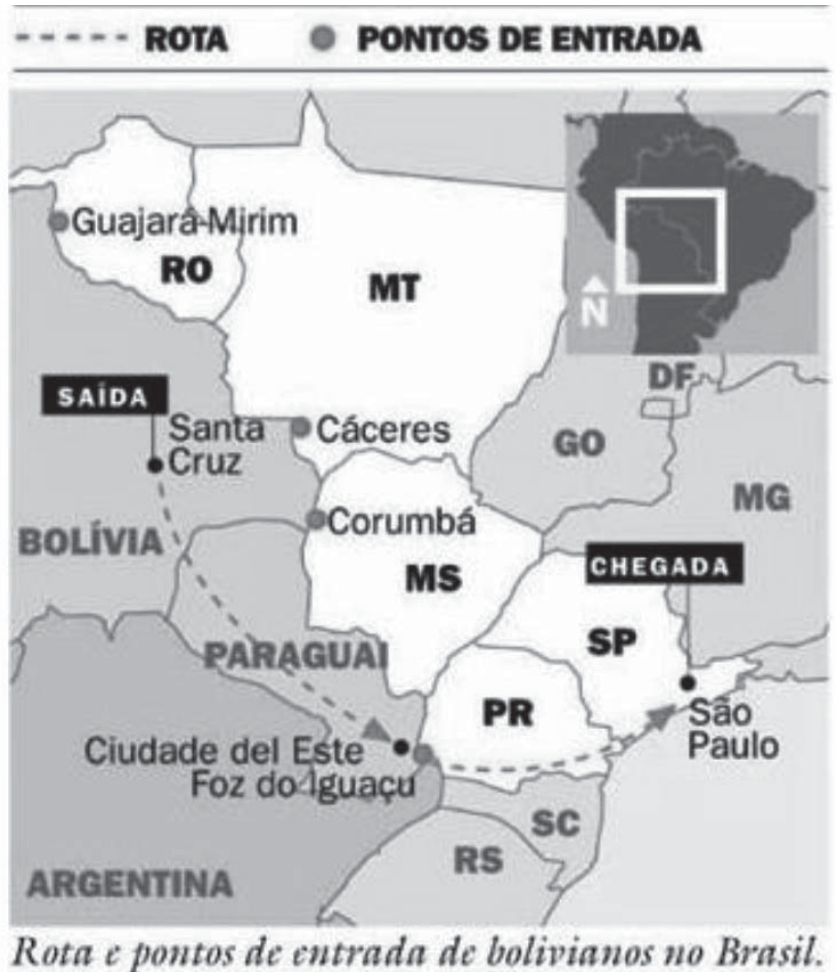

Mapa 2. Inmigrantes bolivianos, según el municipio de residencia, en el año 2000 (en portugués). Fuente: IBGE, Censo demográfico 2000.

(Datos provenientes de documentos obtenidos por medio de la Ley de Acceso a la Información). 
Tabla 3

Diez principales municipios de residencia de los inmigrantes bolivianos

\begin{tabular}{lcc}
\hline Municipio & Número de bolivianos & $\%$ \\
\hline São Paulo & 7.722 & 37,9 \\
Corumbá & 1.098 & 5,4 \\
Guajará-Mirim & 1.077 & 5,3 \\
Rio de Janeiro & 1.020 & 5,0 \\
Porto Velho & 583 & 2,9 \\
Rio Branco & 507 & 2,5 \\
Campo Grande & 388 & 1,9 \\
Guarulhos & 367 & 1,8 \\
Costa Marques & 360 & 1,8 \\
Cuiabá & 283 & 1,4 \\
\hline Subtotal & 13.405 & 65,7 \\
\hline Total & 20.388 & 100,0 \\
\hline
\end{tabular}

Fuente: IBGE, Censo demográfico 2000. (Datos provenientes de documentos obtenidos por medio de la Ley de Acceso a la Información).

En el cómputo general de las solicitudes de inmigrantes, São Paulo era el estado que más representaba el otorgamiento de solicitudes, con un total de 9.676 en 2006, 12.057 en 2007, 20.706 en 2008, 18.285 en 2009 y 25.550 en 2010. El estado de Río de Janeiro aparece en segundo lugar, seguido por el estado de Minas Gerais, el estado del Amazonas y el estado del Paraná. Las solicitudes no otorgadas de autorizaciones para el trabajo legal son por no cumplimiento de la exigencia legal en 30 días (41\%), por indicio de la substitución de mano de obra nacional (10\%), pérdida del objeto del contrato laboral (10\%) y otras razones (39\%).

El informe "Perfil Migratorio del Brasil 2009" del MTE divulgó los flujos de la migración internacional hacia Brasil entre 1990 y 2000. Con base en el informe, la inmigración estaría concentrada en las dos principales metrópolis brasileñas: São Paulo y Río de Janeiro (Ministério do Trabalho e Emprego [MTE], Comissão Nacional de População e Desenvolvimento [CNPD], y Organização Internacional para as Migrações [OIM], 2010). Es en la metrópolis de São Paulo que la inmigración internacional de los trabajadores globales y de la migración étnica se concentra: 44\% de los asiáticos, 38\% de 
los japoneses y más de $20 \%$ de los europeos, estadounidenses y argentinos (16\% en este caso) del mismo período.

De acuerdo con el informe, en relación a la ocupación de los 3.866 inmigrantes bolivianos que han identificado sus ocupaciones en el Censo Demográfico de 2000, 4,68\% están en la agricultura y la pesca; $50,75 \%$ en la industria de transformación; 3,7\% en la construcción; 9,44\% en el comercio; 3,55\% en el alojamiento y alimentación; $2,87 \%$ en la intermediación financiera; $2,22 \%$ en la educación; $6,7 \%$ en la salud y en los servicios sociales y $4,63 \%$ en otras actividades (MTE et al., 2010).

Considerando las informaciones oficiales del MTE en relación al número de inmigrantes extranjeros en el Brasil en el período comprendido entre 1960 y 2010, ha aumentado significativamente el ingreso de bolivianos, conforme se observa en la Tabla 4 .

Tabla 4

Cantidad de inmigrantes bolivianos en el Brasil (1960-2010)

\begin{tabular}{cc}
\hline Año & Número \\
\hline 1960 & 8.049 \\
1970 & 1.970 \\
1980 & 12.980 \\
1991 & 15.694 \\
2000 & 11.113 \\
2010 & 27.259 \\
\hline
\end{tabular}

Fuentes: MTE et al., 2010, p. 26; IBGE, 2000'ㅍ IBGE, $2010^{2}$.

\section{Las estimaciones}

Las estimaciones sobre la cantidad de inmigrantes bolivianos son muy controvertidas. Conforme ya señalamos, el Estado brasileño y las esferas administrativas — MTE,

1 Datos provenientes de documentos obtenidos por medio de la Ley de Acceso a la Información.

2 Datos obtenidos por medio de tablas suministradas a través del protocolo 03950003824201313 del Sistema de Acceso a la Información. 
Policía Federal, IBGE- poseen bancos de datos con estadísticas propias y diferentes entre sí, sin comunicación e intercambio de información. No hay, en este sentido, una base única que pueda ser consultada y precisar los números.

Otra cuestión importante de las estimaciones es destacar la reciente preocupación del Estado brasileño por registrar y contar inmigrantes en el país de manera sistemática. De acuerdo con Cymbalista y Xavier:

El real tamaño de la comunidad boliviana en São Paulo presenta una gran variación: el Consulado de Bolivia calcula 50 mil ciudadanos sin documentación, la Pastoral de los Inmigrantes piensa que habitan 70 mil ciudadanos bolivianos sin documentos en São Paulo, siendo 35 mil solamente en el barrio del Brás; el Ministerio del Trabajo y Empleo tiene una estimación que varía entre 10 y 30 mil ciudadanos sin documentos; el Ministerio Público habla de 200 mil bolivianos en el total (regulares e irregulares), y el Sindicado de las Costureras apunta 80 mil trabajadores irregulares (lo que incluye familias brasileñas y bolivianas). El único consenso entre estas estimaciones es el hecho de que São Paulo abriga el mayor número de inmigrantes bolivianos en el Brasil [traducción del autor] (2007, p. 123).

Los datos más recientes del MTE se obtienen a partir del libro Perfil Migratório do Brasil 2009. Los que presenta la Tabla 5 se refieren a la totalidad, independiente de si están en la categoría de trabajadores o refugiados políticos, junto a los datos del IBGE para el Censo de 2010 de la Policía Federal para 2013, retratando los mayores grupos de nacionales sudamericanos que viven en Brasil: 
Tabla 5

Extranjeros sudamericanos en Brasil en 1970, 1980, 1991, 2000, 2010 y 2013

\begin{tabular}{ccccccccccccccc}
\hline País & \multicolumn{2}{c}{ Argentina } & \multicolumn{2}{c}{ Bolivia } & \multicolumn{2}{c}{ Chile } & \multicolumn{2}{c}{ Paraguay } & \multicolumn{2}{c}{ Uruguay } & \multicolumn{2}{c}{ Perú } & \multicolumn{2}{c}{ Total } \\
\hline Año & $\mathrm{N}^{\circ}$ & $\%$ & $\mathrm{~N}^{\circ}$ & $\%$ & $\mathrm{~N}^{\circ}$ & $\%$ & $\mathrm{~N}^{\circ}$ & $\%$ & $\mathrm{~N}^{\circ}$ & $\%$ & $\mathrm{~N}^{\circ}$ & $\%$ & $\mathrm{~N}^{\circ}$ \\
\hline 1970 & 17.213 & 26,14 & 10.712 & 16,27 & 1.900 & 2,89 & 20.025 & 30,41 & 13.582 & 20,63 & 2.410 & 3,66 & 65.842 \\
1980 & 26.633 & 26,63 & 12.980 & 12,98 & 17.830 & 17,82 & 17.560 & 17,55 & 21.238 & 21,23 & 3.789 & 3,79 & 100.030 \\
1991 & 25.468 & 23,45 & 15.694 & 14,45 & 20.437 & 18,82 & 19.018 & 17,51 & 22.141 & 20,39 & 5.833 & 5,37 & 108.591 \\
2000 & 27.531 & 21,27 & 20.388 & 15,75 & 17.131 & 13,24 & 28.822 & 22,27 & 24.740 & 19,12 & 10.814 & 8,36 & 129.426 \\
2010 & 16.381 & 14,93 & 27.259 & 24,84 & 5.128 & 4,67 & 44.335 & 40,40 & 9.016 & 8,22 & 7.616 & 6,94 & 109.735 \\
2013 & 50.322 & 21,31 & 76.460 & 32,38 & 28.392 & 12,03 & 23.105 & 9,79 & 33.823 & 14,33 & 23.997 & 10,16 & 236.099 \\
\hline
\end{tabular}

Fuentes: MTE et al., 2010; IBGE, 2010³; Policía Federal ${ }^{4}$.

Es importante observar el significativo crecimiento del ingreso de inmigrantes bolivianos en Brasil a lo largo de estos censos demográficos, siendo el segundo grupo de menor expresión en 1980 y alcanzando la primera posición en 2013, reforzando aquí las hipótesis ya presentadas a lo largo de este artículo.

\section{Caracterización de los inmigrantes bolivianos}

La caracterización del inmigrante boliviano que llega a Brasil puede ser definida provisoriamente en su mayoría como hombre, joven, soltero y con baja calificación. No obstante, en los últimos años han llegado más mujeres que hombres. Esta disparidad entre los sexos ya fue mayor. Hace 15 años, la población masculina respondía por casi el 74\% del total, porque el movimiento migratorio entre los dos países era un fenómeno eminentemente masculino. En relación a la franja de edad, la mayoría absoluta de estos extranjeros se queda entre los 18 y 44 años, o sea, se encuadra en la población joven y económicamente activa (Barros, 2001).

\footnotetext{
3 Datos obtenidos por medio de tablas suministradas a través del protocolo 03950003824201313 del Sistema de Acceso a la Información.

4 Datos obtenidos por medio del Memorándum No 324/2013-SNJ/MJ del Ministerio de la Justicia.
} 
Paradojalmente, si ellos vienen a Brasil creyendo dejar atrás la pobreza y la economía de subsistencia, cuando llegan a nuestro país son sometidos a mayores dificultades y servicios pesados; trabajo informal o exceso de trabajo; falta de la calificación profesional para la mayoría y, para otros, a pesar de poseer calificación en el país de origen, la contingencia de sujetarse a cualquier trabajo; remesas monetarias de los migrantes al país de origen bajo altas tasas de transferencia; dificultad del manejo de las ganancias sea por carencia de formación o por indefinición del local de inversión; trabajos altamente rotatorios (Hirsch, 2008).

Además, el migrante, dejando su tierra, quiebra su red originaria de relaciones para reconstituirla en otro lugar. Es por este motivo que, cuando se disloca solo o con la familia, intuitivamente busca lugares donde están sus compatriotas o conocidos. Innumerables son los desafíos de orden social y cultural enfrentados por el inmigrante, conforme se puede visualizar en el Cuadro 1. 
Cuadro 1

Desafíos sociales y culturales enfrentados por los inmigrantes (adaptado)

\section{Desafíos sociales}

- Confrontación con actitudes racistas de intolerancia y xenofobia;

- Ruptura de las raíces familiares;

- Acogida como fuerza de trabajo y no como ciudadano internacional;

- No es tratado como ciudadano, sino como intruso;

- Remuneración incompatible con su trabajo o con el resultado de su trabajo;

- Relativización por su diversidad;

- Esfuerzo para escapar de las redes de explotación en sus más variados tipos;

- Migraciones internacionales cada vez más femeninas;

- Acusación de ser un elemento de desequilibrio en las remuneraciones legales por aceptar el trabajo por cualquier precio.

\section{Desafíos culturales}

- Barreras lingüísticas y culturales del país receptor;

- Dificultad de inserción en la cultura local;

- Choque de generaciones en el contexto familiar;

- Dificultad de convivencia con las diferentes visiones del mundo;

- Aprendizaje del nuevo idioma sin el conocimiento de las raíces culturales que lo constituyen;

- Riqueza de la cultura del migrante muchas veces es explorada folclóricamente en los países de emigración;

- Desligamiento de la evolución de la realidad social, económica, política y cultura del país de origen.

Fuente: Zamberlam et al. (2009).

Claramente existen otros desafíos que los migrantes internacionales deberán sortear, principalmente en lo que respecta a las esferas jurídicas y religiosas. Al mismo tiem- 
po en que los capitales y mercaderías tienen libre circulación, los migrantes encuentran barreras cada vez más imposibles de superar. Así, el proceso de globalización se revela excluyente, asimétrico y paradojal. La concentración de la riqueza y de la renta en los países centrales (o desarrollados, según el punto de vista del lector) también condensa las oportunidades de trabajo, llevando al crecimiento del flujo de los países más pobres en dirección a los países más ricos, o sea, desde Asia, África, Latinoamérica y el este europeo hasta Europa Central, Estados Unidos y Japón (Zamberlam et al., 2009).

\section{Los bolivianos y el trabajo ilegal, degradante y esclavo}

Los bolivianos dejan Bolivia por varias razones, aunque las económicas toman mayor fuerza a la hora de emigrar. Según Silva (2006), el mercado del trabajo brasileño, incluso en la denominada "década perdida", o sea, 1980, ofrecía más oportunidades de empleo que el mercado de trabajo boliviano, atravesado por una profunda crisis económica, con altos índices de inflación y desempleo.

Enviar recursos para la familia que ha quedado en Bolivia parece estar en el horizonte de los inmigrantes bolivianos que aquí llegan en busca de trabajo. Así podemos preguntarnos hasta qué punto estos inmigrantes tendrían alguna perspectiva de quedarse definitivamente en Brasil.

Investigaciones indican que existe una diferenciación de expectativas entre aquellos que están hace más tiempo en el país y aquellos que han llegado recientemente. Para los primeros, regresar a Bolivia es una posibilidad, pero ya no se pone más como algo a ser intentado en el corto plazo en razón de los lazos que han sido creados con Brasil. Para los recién llegados, el regreso es algo más objetivo, pues la razón de su inmigración es la conquista de recursos para realizar el sueño de lograr independencia económica en el país de origen (Silva, 2006).

Las redes sociales actúan intensamente junto a los inmigrantes y les ayudan a encontrar trabajo, conforme muestra la investigación de Hirsch (2008). Para esta autora, "es frecuente en las comunidades de los inmigrantes que haya una asociación entre las nacionalidades y el tipo de actuación profesional. Un ejemplo son los inmigrantes bolivianos en São Paulo, que actúan mayoritariamente en la confección" [traducción de los autores] (Hirsch, 2008, p. 2). 
El reclutamiento de estos bolivianos se hace, muchas veces, por medio de anuncios en las radios locales, que prometen salarios con alimentación y alojamientos incluidos (Hirsch, 2008). En otros casos, el contacto es hecho directamente por el empleador que, según Hirsch, no es raro que lleve toda la familia del inmigrante a Brasil.

El "reclutamiento" en el local donde vive el potencial inmigrante no es un fenómeno desconocido para los brasileños. A comienzo del siglo XX las plantaciones de café exigían grandes cantidades de mano de obra y las industrias brasileñas necesitaban de mano de obra más especializada. La solución encontrada para estas dos coyunturas fue la importación. El Estado brasileño ha realizado intensas campañas de propaganda en países estratégicos como Italia, Alemania y España, prometiendo una buena vida y buenas condiciones de trabajo y vivienda. Pero tales promesas resultaban falsas, pues cuando los inmigrantes llegaban a Brasil descubrían que ya tenían una deuda por la compra del pasaje. En los primeros tiempos, el salario era apenas destinado al pago del pasaje, de la vivienda y la alimentación. La condición de endeudados continuaba, muchas veces, por años.

La inmigración boliviana, en cierta medida, reedita esta experiencia de la exploración del trabajo del inmigrante, siendo el reclutamiento hecho en Bolivia y con promesas de buena vida en Brasil. La diferencia, tal vez más nítida, es el hecho de que hace 100 años atrás se efectuó con el permiso del Estado brasileño, aunque no explícitamente. Hoy el Estado combate este tipo usual de práctica ejercido por dueños de fábricas de tejidos de São Paulo (principalmente).

La actividad que más utiliza mano de obra boliviana indocumentada es la confección. Para Silva (2009), una explicación es la cuestión de las facilidades de inserción del inmigrante boliviano en este mercado de costura, el cual no exige experiencia, límite mínimo para la actividad, y la lengua no es un obstáculo. En cuanto al empleador, más allá de estos elementos existe la facilidad de burlar la cuestión salarial. Como el salario está ligado a la cantidad de cortes producidos, hay total libertad para el empleador en definir el precio de la fuerza de trabajo de estos inmigrantes. Las confecciones que utilizan el trabajo boliviano en su mayoría son irregulares y se ubican en lugares lúgubres, en sótanos, sin ventanas, iluminación no adecuada y, en algunos casos, con ausencia de buenas condiciones de higiene.

El trabajo degradante es una rutina para los inmigrantes bolivianos que viven en São Paulo, y el Estado brasileño no es eficaz en terminar esta situación. Según la superintendente regional del Trabajo y Empleo, Lucíola Rodrigues Jaime, el problema "no 
es solamente laboral, sino de inmigración y social; todas las entidades, todos nosotros, que trabajamos con eso, sabemos de la difícil situación de los inmigrantes en São Paulo y somos incapaces, actuando aisladamente, de resolver el problema" [traducción de los autores] (Asesoría de Comunicación de la SRTE/SP, comunicación personal, 30 de enero de 2008).

Las iniciativas oficiales creadas para contener el trabajo degradante del inmigrante boliviano, como la Guía de Promoción del Trabajo Decente para Extranjeros, la primera Conferencia Nacional del Empleo y del Trabajo Decente en el Brasil, el Pacto Municipal de Tres Partes Contra el Fraude y la Precarización, y por el Empleo y Trabajo Decentes en São Paulo, destacan la situación trágica y casi incontrolable de precarización en el trabajo; pésima calidad de vida y de trabajo; ausencia de criterios mínimos de legislación, seguridad y salud de los locales de trabajo; ausencia de responsabilidad solidaria, en los casos de outsourcing, de los tomadores de servicios, en casos de no observancia de la legislación laboral u otras irregularidades; $y$, finalmente, la falta de una entidad independiente para el monitoreo de la condición del migrante en la ciudad y proveer las comunidades extranjeras de informaciones (Camargo, 2008).

El trabajo degradante que los inmigrantes enfrentan, en algunos casos, puede ser definido como trabajo esclavo y tanto el Estado como la sociedad brasileña saben de la ocurrencia de tal situación. El estudio de la Secretaria de Inspección del Trabajo (SIT) y del MTE muestra que desde la creación del Grupo Especial de Fiscalización Móvil, en 1995, fueron rescatados en Brasil 38.769 trabajadores en situación análoga a la esclavitud. Entre 1995 y 2002 hubo 5.893 rescates. Entre 2003 y 2010 hubo 32.986 (datos provenientes de documentos obtenidos por medio de la Ley de Acceso a la Información).

El combate al trabajo esclavo en Brasil es hecho comúnmente por el Legislativo brasileño y por las organizaciones no-gubernamentales. Es un combate complejo con dificultades de obtener informaciones y reunir en registros fidedignos, además, en muchos casos, peligroso, principalmente si el combate quedase en las áreas rurales de Brasil, cuya práctica es el asesinato de personas que ejercen la fiscalización de este tipo de exploración.

Oficialmente se garantiza algún recurso para el trabajador cuando es liberado de la condición análoga a la de esclavo con la creación del Seguro de Desempleo Especial para el Refugiado (Casa Civil, Subchefia para Assuntos Jurídicos, 2002), que entrega el valor de un salario mínimo para cada brasileño o extranjero en esta situación. 
Desde 1995, el gobierno brasileño liberó a casi 40.000 personas del trabajo esclavo. Esta forma de súper-explotación puede ser encontrada en las cadenas productivas de carne, acero, algodón, soja, maíz, arroz, judías, frutas, madera, yerba mate, caña de azúcar, cacao, babasu, entre otras (Sakamoto, 2010).

Tales iniciativas, sin embargo, no están relacionadas con el trabajo de los inmigrantes en general y de los bolivianos en especial. En las acciones de fiscalización del trabajo esclavo entre los brasileños, la cuestión del trabajo esclavo del inmigrante apenas aparece. Lamentablemente, todavía no hay estadísticas oficiales para el caso de los inmigrantes en relación a los trabajos forzados, esclavo o ilegal, aunque el MTE sabe de su existencia.

Las informaciones sobre el trabajo esclavo de bolivianos en Brasil se saben por medio de denuncias y reportajes en la televisión y periódicos. Raramente son hechas por el propio inmigrante boliviano, en vista de su temor a ser repatriado. En tales reportajes, las historias se repiten; todas presentan características como lugares insalubres para los trabajadores, falta de remuneraciones, exceso de horas trabajadas - entre 14 y 16 horas por día (mientras la jornada de trabajo en Brasil es de seis u ocho horas diarias) - e incluso la muerte, como ocurrió con el niño Brayan en la ciudad de San Pablo, mostrando la gravedad de las condiciones en que estos inmigrantes bolivianos viven en Brasil.

Son historias que ocurren en diversos lugares del país, no solo en São Paulo. En octubre de 2010 fueron encontrados 15 inmigrantes bolivianos sometidos a una situación análoga a la esclavitud. La empresa F.G. Indústria e Comércio de Uniformes e Tecidos Ltda. del Estado del Paraná ganó una licitación de $\mathrm{R} \$ 4,3$ millones para la producción de 230.000 chalecos para el Censo 2010 del IBGE. A pesar del edicto de no autorizar el outsourcing, la empresa ganadora ha hecho este proceso en casi toda su producción $(99,12 \%)$. Una de las empresas subcontratadas por la industria ganadora de la licitación, repasó parte de su encomienda para una tercera empresa: un taller de costura precaria en São Paulo, donde el MTE encontró trabajadores bolivianos en situación de esclavitud.

Las acciones de fiscalización en las grandes ciudades, según el propio MTE, han encontrado efecto en algunas empresas de cobertura nacional que firmaron el compromiso de cancelar pedidos de proveedores que emplean mano de obra irregular.

Este compromiso, firmado en 2007 entre las grandes tiendas de vestuario del Brasil -Renner, Marisa, C\&A y Riachuelo- y el Ministerio Publico del Trabajo de São Paulo, podría indicar disposición por parte de los gobiernos y empleadores hacia el combate 
de la inmigración indocumentada y la esclavitud de los inmigrantes, pero no es lo que parece. Después de seis años de firmados estos compromisos, el uso del trabajo degradante de los inmigrantes, sobre todo los bolivianos, aún existe y es cada vez mayor.

El propio coordinador general de Inmigración de Brasil (CGIg), Paulo Sérgio de Almeida, en 2007, afirmaba que el MTE recibía denuncias de explotación del trabajo de inmigrantes bolivianos, incluso tras el compromiso firmado entre las tiendas y el Ministerio Público. En el 2008 la situación continuaba igual, según el coordinador:

En las conversaciones de este viernes, descubrimos que los bolivianos que trabajan irregularmente en estas confecciones son llevados para otras ciudades, ya que la acción fiscal de la Secretaria de Inspección del Trabajo del MTE está cercando los exploradores, de acuerdo con su declaración. Así, nosotros conseguimos inclusive ayudar la fiscalización de la SIT y ayudar el trabajador que es explotado. La condición de irregularidad en el país empeora esta explotación [traducción de los autores] (Conselho da Comunidade Luso-Brasileira do Estado de São Paulo, 2008).

Más allá del Estado vía MTE, principalmente, otras instituciones brasileñas se organizan para combatir el trabajo degradante del inmigrante boliviano. Es el caso del Servicio Pastoral de los Inmigrantes y del Centro de Apoyo al Inmigrante de la Iglesia Católica, que discuten la formación de cooperativas para prestar servicio a los proveedores y vendedores. Cerca de 150 personas en situación legal en el país ya están registradas para formar las cooperativas.

\section{Los refugiados políticos y las cuestiones culturales todavía serían categorías residuales}

La característica de los refugiados bolivianos no favorece el levantamiento de datos precisos, puesto que muchos ingresan a Brasil vía la frontera del estado de Rondônia, se concentran en la ciudad de Guajará-Mirim y se mantienen irregulares por decisión propia. Otros optan por regularizar su situación. Esto implica accionar el área diplomática y política del Brasil. Es posible tener nociones en términos de estimativas o incluso de las situaciones de estos refugiados mediante entrevistas, reportajes de periódicos y noticias de la prensa, por ejemplo. 
En una entrevista a un periódico brasileño, la religiosa hermana Rosita Milesi, cuando habló sobre el ingreso de refugiados haitianos, relató que, representando al Instituto de Migraciones y Derechos Humanos (IMDH), juntamente con los miembros del Gobierno Federal y del Alto Comisionado de las Naciones Unidas para los Refugiados (ACNUR), integró una comisión que estuvo en el estado del Acre, donde se concentran millares de refugiados haitianos. Los refugiados bolivianos que se encuentran en la región cedieron parte de su estructura para recibir a los recién llegados refugiados haitianos (MTE, Conselho Nacional de Imigração, 2011).

El número de refugiados bolivianos registrado por el Comité Nacional para los Refugiados del Ministerio de Justicia brasileño llega a 127 ciudadanos con estatus de refugiados, así divididas las concesiones de refugio por año: 112 bolivianos reconocidos en 2009, tres en 2010, uno en 2011 y siete en 2012 (datos provenientes de documentos obtenidos por medio de la Ley de Acceso a la Información). Probablemente estos números tienden a aumentar, dada la fuerza política y el apoyo popular que el gobierno de Evo Morales está adquiriendo con el tiempo, eliminando cada vez más las posibilidades de acción de la oposición.

Como ya lo señalamos en este artículo, la cuestión de la inmigración boliviana está ligada a la situación interna de Bolivia, sea en términos económicos o políticos. La cuestión de los refugiados políticos, obviamente, está relacionada a los aspectos y coyunturas políticas del país. En noticias de periódicos, se difundió el conocimiento de cómo los bolivianos reaccionan frente a los conflictos políticos internos en Bolivia en relación a la posibilidad de salir del país.

De acuerdo con estos reportajes, en el período de conflicto (septiembre de 2008), entre los partidarios del presidente Evo Morales y la oposición, en la ciudad fronteriza de Pando,

los bolivianos atravesaron la frontera en septiembre del año pasado, cuando la región de Pando fue panorama de violentos enfrentamientos entre los opositores y partidarios del gobierno del presidente Evo Morales. En los disturbios, cerca de 20 personas murieron y otras 70 resultaron heridas (UOLNotícias, 2009).

Según informaciones oficiales, los solicitantes beneficiados con el estatus de refugiado por el Comité Nacional para los Refugiados (CONARE) se quedan en la ciudad de Brasileia, en el estado del Acre, ciudad fronteriza con la región de Pando y donde, en 
medio de los disturbios, el periódico en línea UOLNotícias (2009) dice creer que llegaron más de 1.000 bolivianos, de los cuales la mayoría ya retornó a su país.

La misma idea de que cuando hay tensiones políticas en Bolivia, los bolivianos se aprovechan de la proximidad fronteriza con el Brasil para refugiarse momentáneamente o incluso para obtener el estatus de refugiados políticos. De acuerdo con Otávio Cabral, quien describe la situación de Bolivia en la frontera en la ciudad de Cobija:

Solamente en el día 15 de septiembre, cuando el Ejército fue hacia las calles, 400 bolivianos cruzaron el Puente Wilson Pinheiro, inaugurada por el presidente Lula en 2005, y llegaron a la ciudad de Brasileia, donde se quedaron por la noche, sin abrigo, en la plaza central de la ciudad. En los días siguientes, más de 600 bolivianos llegaron al Brasil por los puentes, nadando por el río Acre o caminando por la selva. Hoy, el mayor grupo de refugiados está abrigado en el gimnasio de deportes, llamado por los bolivianos de "coliseo". El restante se queda en hoteles, posadas, casas de amigos y sedes de sindicatos. Todos reciben alimento y asistencia médica del gobierno del Acre y son protegidos por el Ejército brasileño. Los refugiados pasan el día oyendo las radios y canales de televisión bolivianos en busca de noticias, principalmente sobre la situación de Leopoldo Fernández, que sigue capturado en un cuartel del Ejército en La Paz a pesar de que la Suprema Corte le concediera un habeas corpus para que fuera puesto en libertad (Cabral, 2008, p. 1).

El reportaje de este periodista es interesante pues nos permite tener una noción de las condiciones políticas para que Brasil pueda involucrarse en la situación política interna y externa de las relaciones con Bolivia. Cuando describimos las particularidades de la frontera de Brasil con Bolivia, es posible verificar que:

Hay dos grupos de oposicionistas bolivianos en Brasileia y en Epitacolândia. En hoteles, casas alquiladas y viviendas de familiares, se quedan los profesionales liberales ligados directamente a Fernández, que son dirigentes del partido de oposición a Morales. Es el caso del abogado Carlos Soarez, amigo de infancia de Leopoldo, que cerró su estudio en Cobija, cruzó el puente en un microbús y se alojó en el mejor hotel de la región. "Si yo cruzo la frontera ahora, durante el estado de sitio, seré capturado. Tenemos que esperar que el Ejército salga de las calles y Leopoldo de la cárcel para nosotros reorganizar la oposición al dictador cocalero", grita Soarez en el balcón del hotel, con periódicos y revistas bolivianos sobre las piernas. Ese grupo, según las informaciones de la Policía Federal, estaría usando haciendas en el territorio brasileño para organizar la resistencia, lo que es también ilegal. La principal líder del grupo es Ana Melena de Suzuki, presidenta del Comité Cívico de Pando. Acusada formalmente por la policía boliviana de ser responsable por la masacre, ella 
también huyó para Brasileia. En territorio brasileño también fue perseguida por agentes bolivianos y pidió ayuda a la Policía Federal brasileña, que la llevó más allá de la frontera. Buena parte de los líderes autonomistas ya solicitó refugio político al Brasil. Ellos temen ser perseguidos y capturados por la masacre si vuelven a Bolivia. El periodista Jairo Vallejos, 24 años, por ejemplo, filmó un tiroteo entre militares y autonomistas en el aeropuerto de Cobija, el día siguiente a la masacre. Perseguido, huyó a Brasil con su esposa, embarazada de nueve meses, que tuvo su primera hija en el hospital de Brasileia (Cabral, 2008, p. 1).

La situación es, por lo tanto, compleja, y demanda atención por parte de las autoridades locales brasileñas y del Gobierno Federal, puesto que afecta las cuestiones diplomáticas. En el caso descrito, particularmente, según el representante del ACNUR en Brasil, Javier Lopez-Cifuentes, "los refugiados son personas de clase media, con un estilo social mayor". No hay un estilo de comportamiento por parte de estos refugiados "circunstanciales". Ellos ingresan en el Brasil, se alojan como pueden y esperan pasar las tensiones. Algunos de ellos se registraron en la Defensa Civil de Acre en busca de protección. Pero, por otra parte, como afirma el reportaje, "la oposición huye e intenta hacer la resistencia armada en el Brasil" (Cabral, 2008).

Si no podemos saber la cantidad o incluso el perfil exacto de los refugiados políticos bolivianos, en algunos casos excepcionales, la identidad acaba por ser revelada. En reconocimiento del gobierno brasileño como refugiado político, el juez Luis Tapia Pachi alega ser víctima de persecución política por el gobierno del presidente Evo Morales. Por unanimidad, aun en diciembre del año pasado, el CONARE, órgano vinculado al Ministerio de Justicia de Brasil, reconoció que Tapia debería ser acogido en el país como refugiado político. Por ser un proceso sigiloso, la decisión no fue divulgada en ese período, esto es, en 2010.

El juez boliviano era responsable por la investigación de un supuesto complot de la oposición para matar al presidente Evo Morales, en abril de 2009. Durante las investigaciones, la policía boliviana invadió un cuarto de hotel, en Santa Cruz de La Sierra, y mató a los tres sospechosos. Mientras el gobierno afirmaba ser una operación bien exitosa para evitar un atentado contra el Presidente, los opositores decían que las muertes eran un exceso de la policía. Tapia afirmó que comenzó a ser perseguido por no aceptar la jurisdicción del caso de Santa Cruz de La Sierra para la capital del país, La Paz, y que había sufrido amenaza de muerte. Dijo que podría ser capturado bajo orden del Ministerio Público boliviano (Recondo, 2011). 
En septiembre de 2013, otro caso de fuga de un senador boliviano a territorio brasileño obtuvo gran repercusión en la prensa sudamericana y ocasionó incluso la salida del embajador Antonio Patriota del cargo de ministro de Relaciones Exteriores de Brasil. Roger Pinto Molina es uno de los principales parlamentarios de oposición al gobierno del presidente Evo Morales. Alegando persecución política, desde el 8 de junio de 2012 habitaba en la embajada brasileña en La Paz en condición de asilado. Según el gobierno boliviano, el pedido de asilo fue hecho para no responder a la justicia por los crímenes de daño económico al Estado, calculados en US\$1,7 millones.

Para llegar a Brasil, Pinto Molina salió en coche desde La Paz y siguió hasta Corumbá, en el Estado de Mato Grosso do Sul. El recorrido de más de 20 horas fue hecho por un coche de la embajada brasileña. La autorización fue concedida por el jefe de Cancillería, el ministro Eduardo Saboia, que sustituía temporalmente al embajador Marcelo Biato. Desde Corumbá, Molina siguió hasta Brasilia por avión.

Un día después de la fuga del senador boliviano, la ministra de Comunicaciones de Bolivia, Amanda Davila, dijo que el caso no afectaría las relaciones con Brasil:

Bolivia y las relaciones con Brasil se mantienen en situación de absoluta cordialidad y respecto. El gobierno boliviano y el Presidente Evo Morales siempre manifestaron todo su cariño y respecto a la Presidenta Dilma Rousseff y al gobierno brasileño (Richard, 2013).

No obstante, las relaciones entre Brasil y Bolivia prometen ser tema del orden bilateral entre los dos países, además de la cuestión de los inmigrantes bolivianos, en el encuentro presidencial entre los presidentes Morales y Rousseff previsto para fines de 2013.

\section{Conclusiones}

Discutimos con aportes y elementos teórico-empíricos la situación de los inmigrantes bolivianos, sus formas de enfrentar las dificultades propias de la situación de inmigración y la actuación del Estado brasileño frente a una realidad de penuria y explotación del trabajo de esta población que llega al país. Intentamos mostrar que el flujo de bolivianos hacia Brasil es creciente, como lo indican los estudios de especialistas en el tema, aunque la actuación del Estado brasileño no responde con efectividad para el control de las explotaciones de los trabajadores bolivianos. ¿Cuál es la explicación para tal 
debilidad del Estado brasileño? Podemos, en una primera instancia, destacar la debilidad burocrática.

Así que el tema de la inmigración expone una paradoja en la investigación social, pues es difícil lograr conciliar la teoría con los datos empíricos. Enfrentamos esta dificultad a lo largo de este artículo, una vez que el fenómeno de la inmigración boliviana es un proceso que está en curso y no tiene perspectivas de un final próximo. En otras palabras, corremos el riesgo de escribir un texto obsoleto. Para huir de esta obsolescencia natural de un análisis datado históricamente, centramos la investigación en las características generales de esta inmigración y las ilustramos con los datos disponibles.

Es importante señalar las dificultades culturales que los inmigrantes bolivianos deben enfrentar. También deseamos señalar, sobre todo, las razones de esta inmigración que, en este caso, son todavía resultado de las dificultades propias del país de origen, en términos de condiciones de vida, por lo que acaban por salir en busca de trabajo, cualquiera sea.

En este texto presentamos datos y cuestiones sobre la inmigración boliviana en Brasil, destacamos la necesidad de mejorar los datos oficiales y resaltamos el temor de los inmigrantes - sobre todo los indocumentados- de hacerse visibles al poder público, por temor a las sanciones que puedan sufrir en virtud de su condición indocumentada. Sin embargo, manejar los datos disponibles es útil para reflexionar sobre la debilidad del Estado brasileño en cuanto al tema de la inmigración. Este se muestra débil en las posibilidades de contener y castigar las explotaciones que los inmigrantes bolivianos sufren. Se puede argumentar que si ya es difícil combatir el trabajo esclavo de los brasileños, cuando se trata de los extranjeros las dificultades son mayores, pues se añaden cuestiones como el temor de hacer la denuncia y generar la expulsión. De cualquier manera, el grave problema es la recurrencia de estas prácticas por parte de brasileños que usan este tipo de explotación contra trabajadores inmigrantes bolivianos, haitianos y chinos. 


\section{Referencias}

Almeida, P. S. de (Org.) (2008a). Mercosul e as migrações: Os movimentos nas fronteiras e a construção de políticas públicas regionais de integração. Brasília, Brasil: Ministério do Trabalho e Emprego.

-_——. (2008b). Entrevista ao portal do MTE. Recuperado de http://portal.mte.gov.br/imprensa/conselho-nacional-de-imigracao-aprova-resolucao-para-trabalhadores-sulamericanos.htm

Barros, C. J. (1 de octubre de 2001). Senzalas bolivianas. Repórter Brasil. Recuperado de http:// www.reporterbrasil.com.br/exibe.php?id=7

Cabral, O. (17 de noviembre de 2008). Conflito na fronteira. O Noticiário de Imprensa da Aeronáutica (NOTIMP). Recuperado de http://www.fab.mil.br/portal/capa/index.php?d atan=17/11/2008\&page=mostra_notimpol

Camargo, A. P. A. (30 de diciembre de 2008). SRTE/SP propõe pacto contra exploração do trabalhador migrante. Recuperado de http://portal.mte.gov.br/delegacias/sp/srte-sppropoe-pacto-contra-exploracao-do-trabalhador-migrante/palavrachave/srte-sp.htm

Casa Civil, Subchefia para Assuntos Jurídicos. (2002). Lei No 10.608, de 20 de dezembro de 2002. Recuperado de http://www.planalto.gov.br/ccivil_03/leis/2002/L10608.htm

Castles, S. (2004). The factors that make and unmake migration policies. International Migration Review, 38 (3), 852-884.

Castles, S., Miller, M. J. y Ammendola, G. (2005). The age of migration: international population movements in the modern world. Nueva York, Estados Unidos: The Guilford Press.

Conselho da Comunidade Luso-Brasileira do Estado de São Paulo. (2008). Igualdade para todos. Recuperado de http://www.cclb.org.br/noticias/set08/set01_01.htm

Conselho Nacional de Imigração. (1997). Conselho Nacional de Imigração. Recuperado de http://portal.mte.gov.br/cni/conselho-nacional-de-imigracao-cnig.htm

. (2008). Resolução Normativa $N^{o} 79$, de 12 de agosto de 2008. Recuperado de http://portal.mte.gov.br/data/files/FF8080812BA5F4B7012BA73B53803EE5/ rn_20080812_79.pdf 
Cymbalista, R. y Xavier, I. R. (2007). A comunidade boliviana em São Paulo: definindo padrões de territorialidade. Cadernos Metrópole, 17, 119-133.

DalPrá, K. R., Mendes, J. M. R. y Mioto, R. C. T. (2007). O desafio da integração social no MERCOSUL: uma discussão sobre a cidadania e o direito à saúde. Cadernos de Saúde Pública, 23 (Supl. 2), S164-S173. Recuperado de http://www.scielo.br/pdf/csp/v23s2/05.pdf

Hirsch, O. (2008). Migrações Sul-Sul: o caso dos bolivianos no Brasil e na Argentina. Observador On-Line, 3 (4).

Ministério da Previdência Social. (2006). Migrações internacionais e a Previdência Social. Brasília, Brasil: MPAS, SPS, CGEP.

Ministério das Relações Exteriores. (2008). Instrumento executivo entre o Governo da República Federativa do Brasil, o Governo da República da Bolívia e o Escritório Regional para o Cone Sul da Organização Internacional para as Migrações (OIM). Recuperado de http://dai-mre. serpro.gov.br/atos-internacionais/bilaterais/2008/instrumento-executivo-entre-o-governo-da-republica-federativa-do-brasil-o-governo-da-republica-da-bolivia-e-o-escritorioregional-para-o-cone-sul-da-organizacao-internacional-para-as-migracoes-oim/

Ministério do Trabalho e Emprego. (2008). Convenção No 143 sobre os trabalhadores migrantes (OIT, 1975). Recuperado de http://portal.mte.gov.br/cartilha_exterior/convencaon-143-sobre-os-trabalhadores-migrantes-oit-1975.htm

Ministério do Trabalho e Emprego, Comissão Nacional de População e Desenvolvimento (CNPD) y Organização Internacional para as Migrações (OIM). (2010). Perfil Migratório do Brasil 2009. Recuperado de http://www.mte.gov.br/trab_estrang/perfil_migratorio_2009.pdf

Ministério do Trabalho e Emprego, Conselho Nacional de Imigração. (2011). Acta de la I reunión del Ministerio del Trabajo y Empleo: 9 de febrero de 2011. Recuperado de http:// portal.mte.gov.br/data/files/8A7C816A2E7311D1012F0D4ED8812F40/Ata\%20 CNIg\%2009-02-11\%20v4.pdf

Recondo, F. (17 de enero de 2011). Brasil reconhece juiz boliviano como refugiado político. O Estado de São Paulo. Recuperado de http://www.estadao.com.br/noticias/ nacional,brasil-reconhece-juiz-boliviano-como-refugiado-politico,667442,0.htm

Richard, I. (25 de agosto de 2013). Bolívia diz que vinda de senador não afetará relações com Brasil. Portal EBC. Recuperado de http://www.ebc.com.br/noticias/internacional/2013/08/bolivia-diz-que-vinda-de-senador-nao-afetara-relacoes-com-brasil 
Sakamoto, L. (20 de octubre de 2010). Governo encontra escravos produzindo coletes do Censo. Recuperado de http://blogdosakamoto.blogosfera.uol.com.br/2010/10/20/governoencontra-escravos-produzindo-coletes-do-censo/

Sayad, A. (1999). Immigration et "pensée d'Etat". Actes de la Recherche en Sciences Sociales, 129, 5-14.

Silva, C. F. da. (2009). Precisa-se: Bolivianos na Indústria de Confecções em São Paulo. Revista Travessia, 63, 5-11.

Silva, S. da. (2006). Bolivianos em São Paulo: entre o sonho e a realidade. Revista Estudos Avançados, 20, 157-170.

Silva, S. A. da (1999). Hispano-americanos em São Paulo: alcances e limites de um processo de integração. Travessia: Revista do Migrante, 33, 24-32.

Schmidt, B. V. (2013). O Estado e a imaginação sociológica. Revista de Estudos e Pesquisas sobre as Américas, 7 (1), 175-203.

Souchaud, S. y Baeninger, R. (2008). Collas e cambas do outro lado da fronteira: aspectos da distribuição diferenciada da imigração boliviana em Corumbá, Mato Grosso do Sul. Revista Brasileira de Estudos de População, 25, 271-286.

UOL Notícias (7 de junio de 2009). Brasil confirma refúgio a 118 bolivianos que fugiram de Pando. UOL Notícias. Recuperado de http://noticias.uol.com.br/ultnot/ efe/2009/06/07/ult1766u31712.jhtm

Waldman, T. C. (2011). Movimentos migratórios sob a perspectiva do direito à saúde: imigrantes bolivianas em São Paulo. Revista de Direito Sanitário, 12, 90-114.

Weber, M. (2002). Economía y sociedad. Madrid, España: Fondo de Cultura Económica.

_____. (2004). Ciencia e Política: duas vocações. São Paulo, Brasil: Cultrix.

Zamberlam, J. et al. (2009). Desafios das migrações: buscando caminhos. Porto Alegre, Brasil: Sólidus. 